\title{
Unexpected reading dissociation in a Brazilian "nisei" with crossed aphasia
}

\author{
P. Caramelli ${ }^{1,3}$, M.A.M.P. Parente ${ }^{2}$, M.L. Hosogi ${ }^{2}$, M. Bois ${ }^{3}$ and A.R. Lecours ${ }^{3}$ \\ 'Department of Neurology, Hospital das Clínicas, University of São Paulo School of Medicine, \\ 'Speech Pathology School, University of São Paulo School of Medicine, São Paulo, Brazil, and \\ ${ }^{3}$ Laboratoire Théophile-Alajouanine, Centre de recherche, Centre hospitalier Côte-des-Neiges, \\ Montréal, Québec, Canada
}

Correspondence to: P. Caramelli, Rua Itapeva, 518-Suites 601/602, CEP 01332-000, São Paulo $(S P)$, Brazil

\begin{abstract}
There is an increased interest in reading impairments in the Japanese language, due to its particular writing system which includes two different scripts, Kanji (logograms) and Kana (phonograms). Reading dissociations between Kanji and Kana have been described, showing that each system is processed differently by the cerebral hemispheres. We describe the case of a 68 year old Brazilian "nisei" (i.e. born from Japanese parents) who had knowledge of both Japanese and Portuguese. He presented an ischemic stroke affecting the right hemisphere and subsequently developed a Broca's aphasia and an unexpected reading dissociation, with an impairment in Kana reading comprehension and a good performance in Kanji and in Portuguese. These findings suggest that the patient's right and left hemispheres have assumed opposite roles not only for oral but also for written language decodification.
\end{abstract}

Keywords: Brazil - Broca's aphasia - Cerebral dominance - Cerebral infarction - Japan - Reading disability

\section{INTRODUCTION}

Japanese is a unique language since it includes two different writing systems, Kana (phonograms) and Kanji (logograms). Reading impairments in Japanese have been increasingly investigated in the last two decades and several studies have described reading dissociations between Kana and Kanji in aphasic and alexic patients (Sugishita et al., 1992). The elaboration of a functional architecture of reading might explain this pattern (Patterson et al., 1985). According to this model, there are at least two different routes implicated in reading: a phonologically mediated route, used for Kana, and a direct whole-word or lexical based route, used for Kanji (Sasanuma, 1985). Thus, a selective impairment of one of these routes could account for a dissociation between Kana and Kanji reading.

Much debate still exists about the anatomical basis of the Japanese reading process. Recent data suggest that the left hemisphere participates in both Kana and Kanji reading, but that different structures are involved in the process of each writing system (Kawamura et al., 1987; Soma et al., 1989). However, tachistoscopic studies performed in normal subjects have shown that the right hemisphere also intervenes in Kanji reading (Hatta, 1977), while Kana seems to be processed more exclusively by the left hemisphere (Hatta, 1977; Sasanuma et al., 1977). Recently, Sugishita et al. (1992) provided additional data concerning neuroanatomical correlates of Japanese written language, claiming that there is no consistent linkage between the occurrence of reading dissociations and lesion sites.

In the present study, we addressed this question describing a particular reading dissociation found in a bilingual patient presenting an aphasia of the Broca's type, following a right hemispheric stroke. The patient spoke both Japanese and Portuguese. We discuss our findings and suggest some possible factors that could have determined the striking cerebral organization for language observed in this patient. Moreover, the articulation of a functional model for reading allows an interpretation of the clinical picture of an unexpected reading dissociation between three writing systems.

Behavioural Neurology . Vol 7 . 1994 165 


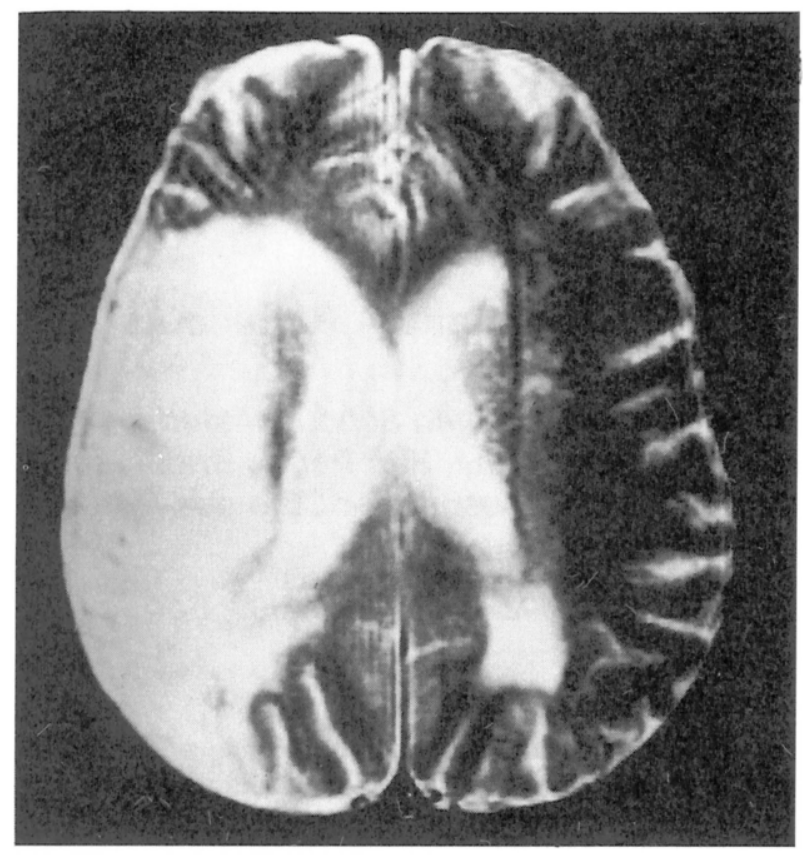

FIG. 1. MRI $T_{2}$-weighted axial view showing a large highintensity area in the territory of the right middle cerebral artery and a smaller, subcortical lesion located posteriorly to the left lateral ventricle.

\section{CASE REPORT}

The patient was a 68 year old right-handed man born in Brazil, called a "nisei" since his parents were Japanese, and who had no family history of left handedness. He studied both Portuguese and Japanese at school (2 years of schooling in Portuguese and 3 years in Japanese). Despite his low educational level, he was a skilled reader, was able to speak both languages at home and at work, and to write fluently in both. His writing skills in Japanese were so good that he became a scribe for his friends and relatives who wanted to send letters to Japan.

In December 1989, he developed left hemiparesis and an aphasia of sudden onset. This clinical picture remained stable until he was first seen in January 1990 in the Hospital das Clínicas of the University of São Paulo School of Medicine, Brazil. At the time of neurological examination, the patient was alert, oriented, and aphasic. His left hemiparesis included the face and was more pronounced in the left upper limb. There were also marked oral and constructional apraxias. There were no signs of anosognosia, prosopagnosia or hemineglect; no visual field deficits were observed on confrontation. A campimetric evaluation was not available.

The computed tomography (CT) scan showed a large low-density area in the distribution of the right middle cerebral artery. Magnetic resonance imaging (MRI) revealed that this ischemic lesion involved the cortical and subcortical aspects of the medial, superior and transversal temporal gyri and of the inferior frontal gyrus, the lateral parts of the precentral and post-central gyri, the inferior parietal lobule and the insula. An older and smaller lesion, located posteriorly to the left lateral ventricle and subcortically distributed, was also identified in the left hemisphere (Fig. 1).

\section{Language evaluation}

Five months later, in May 1990, the patient was submitted to a formal language evaluation using the Portuguese version of the Montréal-Toulouse (MT$86 \beta$ ) Aphasia Battery (Nespoulous et al., 1986). A Japanese adaptation of the battery was also administered. Subtests revealed a good auditory comprehension but a severe deficit in oral production. In matching spoken words to pictures, the patient correctly answered four out of five questions in the Japanese version. He obtained a perfect score, i.e. five out of five in the Portuguese version. He could correctly match pictures to spoken simple sentences and he demonstrated a normal comprehension for simple commands. His auditory comprehension was confirmed to be good by further testing (see below). Nevertheless, he experienced difficulties in matching pictures to long or complex sentences and in comprehending complex commands $(65.6 \%$ of correct responses in the Portuguese version and $60.0 \%$ in the Japanese version).

The spontaneous speech was mainly characterized by unintelligible segments. The patient was unable to produce correct responses in naming tasks. In repetition of Portuguese words and non-words, he presented phonemic paraphasias in $40.4 \%$ of the stimuli and a $44.2 \%$ rate of absence of response. Among the $15.4 \%$ of words correctly produced, there were 10 open-class words, one closed-class, and five neologisms, with an evident word length effect. In repetition tasks of Japanese words and non-words, the patient correctly repeated only $27.9 \%$ of the stimuli (15 open-, two closed-class words and 10 neologisms), also presenting a length effect. Phonemic paraphasias accounted for $51.9 \%$ of the responses and there was a $20.2 \%$ rate of no response.

The patient's performance in reading aloud in Portuguese was extremely poor ( $81.3 \%$ of no response). In $14.9 \%$ of the stimuli, the patient produced phonemic paralexias or he produced the word only after phonemic/semantic facilitators given by the examiner. Only four words $(3.7 \%)$ were correctly read, all of them being open-class and highly frequent words. $\mathrm{He}$ 
was unable to read any non-word. In Kanji reading, only four words were correctly produced. In $52.7 \%$ of the stimuli there was an absence of response and in the remaining $32.6 \%$ of the words either phonemic paralexias occurred or the words were produced following the presentation of phonemic facilitators. In reading aloud Kana stimuli, the patient did not read any of the 72 words and 72 non-words presented to him.

These overall results indicate that the patient showed deficits of the Broca's aphasia type. Finally, a severe constructional apraxia allowed him to draw only simple geometric forms and drastically affected his written output.

Additional matching tasks were devised to further assess his auditory comprehension of both Portuguese and Japanese words. He was also submitted to written language comprehension tests, in Portuguese and Japanese, including Kana and Kanji stimuli. In each test, the patient was requested to point to the correct response. Patient's performance was compared with that of two normal controls, matched for age, languages and education. The first subject (Control 1) was a 58 year old Brazilian "nisei" woman, with 4 years of schooling in Japanese and 12 in Portuguese, who was also a skilled reader and writer in Japanese. The second control was a 63 year old female "nisei", who learned Japanese and Portuguese essentially at home, and was thus able to read and write in both languages, especially in Japanese (she writes letters and reads newspapers regularly). The patient and the controls gave informed consent for all tests. The patient's and controls' results were as follows.

Auditory word and picture-matching task. This task included 50 different line drawings of common objects. The subjects were requested to match an utterance of the examiner with one of four line drawings presented to them on a single display. The patient's score was perfect $(100 \%)$ in Portuguese. He made few errors in Japanese, obtaining a score of $88.0 \%$. Control subjects obtained perfect scores $(100 \%)$ in both languages.

Written word and picture-matching task. This task comprised 50 written words and the same 50 line drawings used previously. The subjects were requested to match a written word with one of four line drawings presented on a single display. In Portuguese, the patient obtained a score of $80.0 \%$ whereas in Japanese, his score was better in Kanji (86.0\%), than in Kana (20.0\%). Control 1 achieved $100 \%$ in Portuguese and in Kanji, and 98\% correct responses in Kana. The second control made no errors in this task $(100 \%$ of correct responses in the three writing systems).

Auditory and written word-matching task. In this task, a card with four written words was presented to the subjects. The examiner read one of the words aloud and the subjects had to select the appropriate word. They were submitted to a total of 80 trials in Kanji and in Portuguese. Targets included an equal number of frequent and non-frequent words, and also an equal number of imageable and nonimageable words, that is, 20 for each type. Kanji frequent words were coded between levels 1 and 4, and non-frequent Kanjis between levels 6 and 8, according to the classification of Kuratami et al. (1982). Portuguese frequent words had a frequency greater than 400 , and non-frequent words a frequency of 200 or less, according to the criteria of Nascimento et al. (1987). The degree of imageability was based on Hogenraad and Orianne's (1981) work. With respect to Kanas, the task comprised an equivalent number of open- and closed-class stimuli. In keeping with the use of the two Kana scripts (Katakana and Hiragana), open-class words were words of foreign origin written in Katakana and closed-class words were written in Hiragana. The number of syllables comprised in the spoken words were controlled. Overall, there was a total of 40 trials.

The patient was correct on $56.2 \%$ of the items in Kanji and on $76.2 \%$ of the items in Portuguese. As for the frequent and imageable words, he recognized $80.0 \%$ of them when they were written in Kanji and $95.0 \%$ when written in Portuguese. On the other hand, the patient could identify neither Katakana writing nor Hiragana writing. His performance attained $12.5 \%$ of correct responses and was totally at random. Both controls performed flawlessly in this task $(100 \%)$.

Auditory and written non-word matching task. Non-words were presented to the subjects, once in Portuguese and once in Hiragana. For each language, there was a total of 45 trials. It is impossible to create non-words in Kanji because Kanji characters do not have separable phonemic components. The subjects were asked to select among four written non-words the one spoken by the examiner. The patient was able to correctly select only $28.9 \%$ in Portuguese and $11.1 \%$ of the non-words in Hiragana. Control 1 obtained a perfect score $(100 \%)$ in Portuguese and in Kana, while Control 2 achieved 95.5\% correct responses in Portuguese and a perfect score $(100 \%)$ in Kana. 


\section{DISCUSSION}

Our patient showed a unique clinical feature of Broca's crossed aphasia associated with an unexpected reading dissociation between three writing systems. He presented serious difficulties in reading Kana words but was able to read Kanji and Portuguese words, at least frequent and imageable ones.

The patient had an ischemic lesion involving the whole territory of the right middle cerebral artery. As mentioned previously, an older and smaller lesion in the left hemisphere was disclosed, therefore, according to the classical definition (Joanette et al., 1982), the term crossed aphasia should be avoided. Since the patient had not been formally tested at that time, we cannot rule out the existence of prior subclinical effects. Nevertheless, there was no evidence of language impairment, including reading difficulties, before the right hemispheric stroke. Furthermore, the language disturbance occurred at the same time as left hemiparesis and, thus, both are closely related.

Hence, the occurrence of a Broca's aphasia suggests that the patient's right hemisphere plays a major role in oral communication. Moreover, some wellknown right hemispheric related neuropsychological deficits, such as hemineglect, prosopagnosia and anosognosia, were not observed. This feature has been previously described in some cases of crossed aphasia, indicating that a mirror image of the usual cerebral organization of cognitive functions may occur (Marshall and Halligan, 1992).

The distribution of the lesions, as shown by MRI, should result in a visual field defect, but no visual field deficits were observed on confrontation, nor did any behavioral evidence of this feature occur. Unfortunately, a campimetric evaluation to explore this possibility was not available. However, we must argue that such a disturbance cannot explain the type of reading dissociation presented by the patient.

Expressive disturbances, as we have found in our patient, are the commonest feature in crossed aphasia (Joanette et al., 1982). Nevertheless, cases presenting Wernicke's crossed aphasia and crossed conduction aphasia in Japanese have also been described (Sakurai et al., 1992; Yamadori et al., 1992). In accordance with Joanette et al.'s (1982) criteria for crossed aphasia, the presence of bilingualism and the knowledge of Kanji must be considered as important factors that could play a role in determining a different cerebral organization for language. Actually, these elements may be responsible for the unusual anatomical pattern of language representation observed in our case. Moreover, the possibility of a prenatal brain injury that could induce a neuroanatomical rearrangement before birth can never be ruled out (Goldman, 1978).

Much debate exists regarding hemispheric participation in the reading process of Japanese language. Some studies on normal subjects suggest that the right hemisphere participates in the processing of logograms, i.e. Kanji (Hatta, 1977), while phonograms (Kana) are processed more exclusively by the left hemisphere (Hatta, 1977; Sasanuma et al., 1977). Other studies state that both Kanji and Kana are treated by the left hemisphere, but different structures are involved in the processing of each writing system (Kawamura et al., 1987; Soma et al., 1989). However, it seems reasonable to assume that even if both writing systems are processed by the left hemisphere, Kanji processing also involves, in some degree, a participation of the right hemisphere.

Impairment of Kana reading has been associated with lesions in the posterior portion of the left inferior temporal lobe and the lateral occipital lobe (Kawahata et al., 1988), the left angular gyrus (Yamadori, 1975), and a left subcortical lesion involving the putamen, the lenticular nucleus, the superior temporal lobe, and the angular gyrus (Hayashi et al., 1985). Hamasaki et al. (1987) reported a case of crossed aphasia due to an hemorrhagic stroke involving the thalamus and the lenticular nucleus which resulted in a better performance in written comprehension of Kanji than Kana. More recently, Yamadori et al. (1992) described a patient with crossed conduction aphasia secondary to a circumscribed lesion in the posterior portion of the superior temporal gyrus and the supramarginal gyrus, presenting the reverse pattern, a superior Kana to Kanji word reading. As far as we know, these two reports are the only ones associating a right hemispheric lesion to a reading dissociation between Kanji and Kana.

Numerous studies have focused on reading dissociations in Japanese non-alphabetic writing systems, but there are few studies reporting dissociations between alphabetic and non-alphabetic codes. Lyman et al. (1938) were among the first to describe such a dissociation. The authors evaluated a bilingual patient (Chinese and English) with a left occipitoparietal tumor accounting for an alexia that was much more marked in Chinese than in English. Their interpretation of the patient's performance was that the whole-word (lexical) reading procedure was impaired which prevented the patient from reading Chinese scripts, whereas a letter-by-letter (phonological) reading strategy, that could not be 
employed in Chinese, enabled him to decipher English words. Since then, other reports about Chinese bilingual patients with crossed aphasia and eventual differences in reading performances have been reported (April and Tse, 1977; April and Han, 1980).

An interesting approach in analysing the reading dissociation presented by our patient is the use of the functional architecture model of reading process (Patterson et al., 1985). According to this model, there are at least two main routes implicated in reading aloud and in reading comprehension: a phonological route that converts orthographic units into phonological units, and a direct route, passing from whole-word orthography to whole-word phonology, accessing the lexicon. Thus, the former is responsible for phonological (or segmental) reading while the latter is implicated in lexical (or global) reading.

Clinical evidence showed that the patient's right hemisphere participated in the processing of Kana as would be expected for a right hemisphere "acting as a left hemisphere". On the other hand, his preserved left hemisphere was able to process the Kanji code which has been related, at least to some degree, to the right hemisphere. Moreover, the clear contrast found between words and non-words in Portuguese suggests that a lexical or global decodification in reading was maintained, while a phonological or segmental decodification was impaired. These two different reading processes may also underlie the dissociation found in the Japanese written codes. As Sasanuma (1985) proposed, the Kana reading implicates a phonological processing, whereas the Kanji code is processed by a direct visual (global) strategy.

Finally, our findings indicate that the patient's left hemisphere intervened in lexical reading, explaining his ability to decode Kanji and Portuguese frequent and imageable words, while his right hemisphere was more involved in phonological processing. Therefore, due to the lesion, our patient was unable to decode the Kana words and also Portuguese non-words. These results suggest that, as for oral language, his left and right hemispheres also assumed the opposite roles for written language decodification.

\section{Acknowledgements}

P. Caramelli holds a scholarship from the $\mathrm{CNPq}$ (Conselho Nacional de Desenvolvimento Científico e Tecnológico), Brazil. This work was supported by grants from FAPESP (Fundação de Amparo à Pesquisa do Estado de São Paulo), Brazil and from the HFSP (Human Frontier Science Program), France.

\section{REFERENCES}

April RS and Han M (1980) Crossed aphasia in a right handed bilingual Chinese man: a second case. Archives of Neurology, 37, 342-346.

April RS and Tse PC (1977) Crossed aphasia in a Chinese bilingual dextral. Archives of Neurology, 34, 766-770.

Goldman PS (1978) Neuronal plasticity in primate telencephalon: anomalous projections induced by prenatal removal of frontal cortex. Science, 202, 768-770.

Hamasaki T, Suzuki K, Hirakawa K, Imahori Y and Nakajima S (1987) Un cas japonais d'aphasie croisée chez un droitier. Revue Neurologique, 143, 47-54.

Hatta T (1977) Recognition of Japanese Kanji in the left and right visual fields. Neuropsychologia, 15, 685688

Hayashi MM, Ulatowska HK and Sasanuma S (1985) Subcortical aphasia with deep dyslexia: a case study of a Japanese patient. Brain and Language, 25, 293-313.

Hogenraad R and Orianne E (1981) Valence d'imagerie de 11300 noms de la langue française parlée. Psychologica Belgica, 20, 21-30.

Joanette Y, Puel M, Nespoulous JL, Rascol A and Lecours AR (1982) Aphasie croisée chez les droitiers I. Revue de la littérature. Revue Neurologique, 138, 575-586.

Kawahata N, Nagata K and Shishido F (1988) Alexia with agraphia due to the left posterior inferior temporal lobe lesion-neuropsychological analysis and its pathogenetic mechanisms. Brain and Language, 33, 296310.

Kawamura M, Hirayama K, Hasegawa K, Takahashi N and Yamaura A (1987) Alexia with agraphia of Kanji (Japanese morphograms). Journal of Neurology, Neurosurgery and Psyhchiatry, 50, 1125-1129.

Kuratami N, Kobayashi A and Okunishi S (1982) A New Dictionary of Kanji Usage. Gakken, Tokyo.

Lyman RS, Kwan ST and Chao WH (1938) Left occipitoparietal brain tumor with observations on alexia and agraphia in Chinese and in English. Chinese Medical Journal, 54, 491-516.

Marshall JC and Halligan PW (1992) Crossed aphasia in a dextral without "minor" hemisphere signs. Behavioural Neurology, 5, 247-250.

Nascimento MFB, Rivenc P and Cruz MLS (1987) Português Fundamental. Instituto Nacional de Investigação Científica, Lisboa.

Nespoulous J-L, Lecours AR, Lafond D, et al. (1986) Protocole Montréal-Tolouse MT-86 d'examen linguistique de l'aphasie-version Beta. Laboratoire Théophile-Alajouanine, Montréal.

Patterson KE, Marshall JC and Coltheart M (1985) Surface Dyslexia. Neuropsychological and Cognitive Studies of Phonological Reading, pp. XXI. Lawrence Erlbaum Associates, London.

Sakurai Y, Kurisaki H, Takeda K, et al. (1992) Japanese crossed aphasia. Neurology, 42, 144-148.

Sasanuma S (1985) Surface dyslexia and dysgraphia: how are they manifested in Japanese? In: Surface Dyslexia. Neuropsychological and Cognitive Studies of Phonological Reading (Eds KE Patterson, JC Marshall and M Coltheart), pp. 225-260. Lawrence Erlbaum Associates, London.

Sasanuma S, Itoh M, Mori K and Kobayashi Y (1977) Tachistoscopic recognition of Kana and Kanji words. Neuropsychologia, 15, 547-553. 
Soma Y, Sugishita M, Kitamura K, Maruyama S and Imanaga H (1989) Lexical agraphia in the Japanese language. Brain, 112, 1549-1561.

Sugishita M, Otomo K, Kabe S and Yunoki K (1992) A critical appraisal of neuropsychological correlates of Japanese ideogram (Kanji) and phonogram (Kana) reading. Brain, 115, 1563-1585.

Yamadori A (1975) Ideogram reading in alexia. Brain, 98, 231-238.
Yamadori A, Yoshida T and Sugiura K (1992) A case of crossed conduction aphasia. Journal of Neurolinguistics, 7, 187-196.

(Received 27 June 1994; accepted as revised 20 October 1994) 


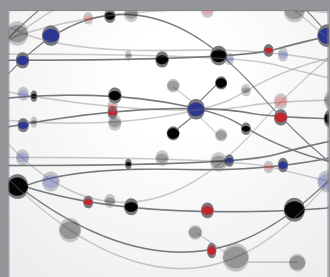

The Scientific World Journal
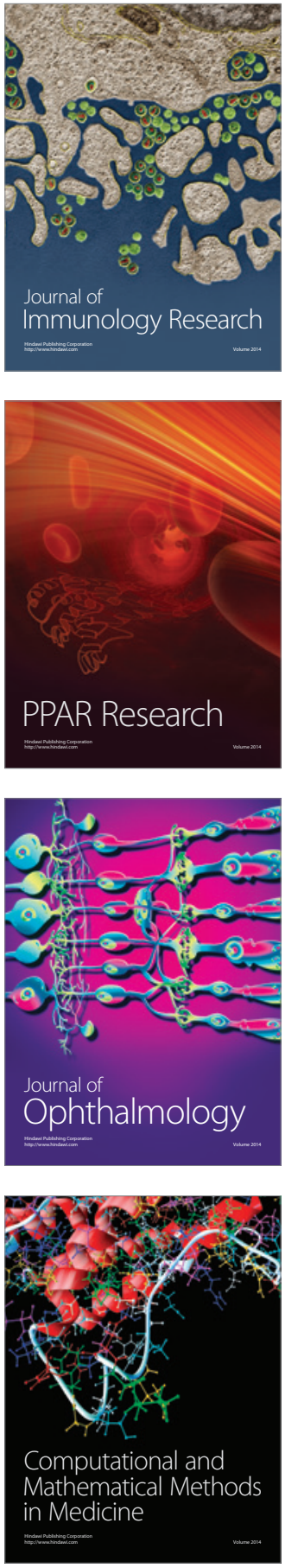

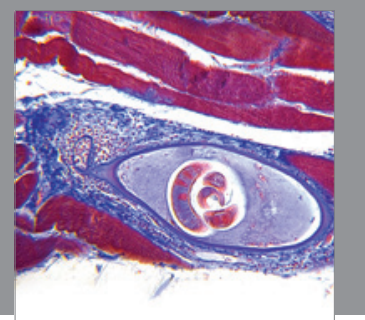

Gastroenterology

Research and Practice
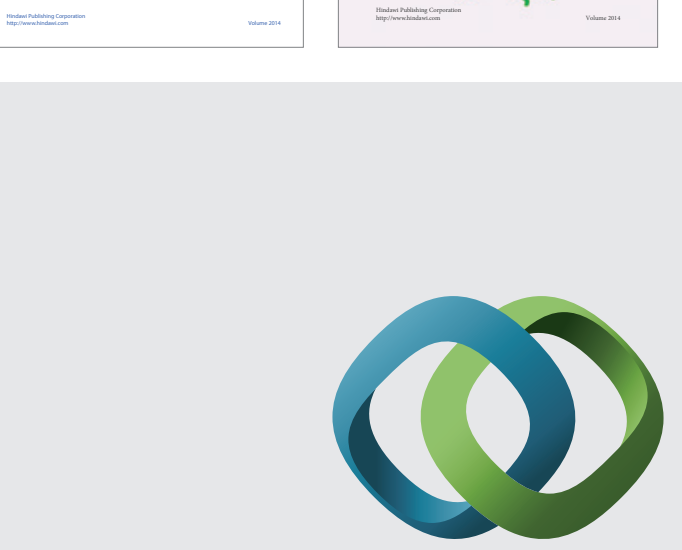

\section{Hindawi}

Submit your manuscripts at

http://www.hindawi.com
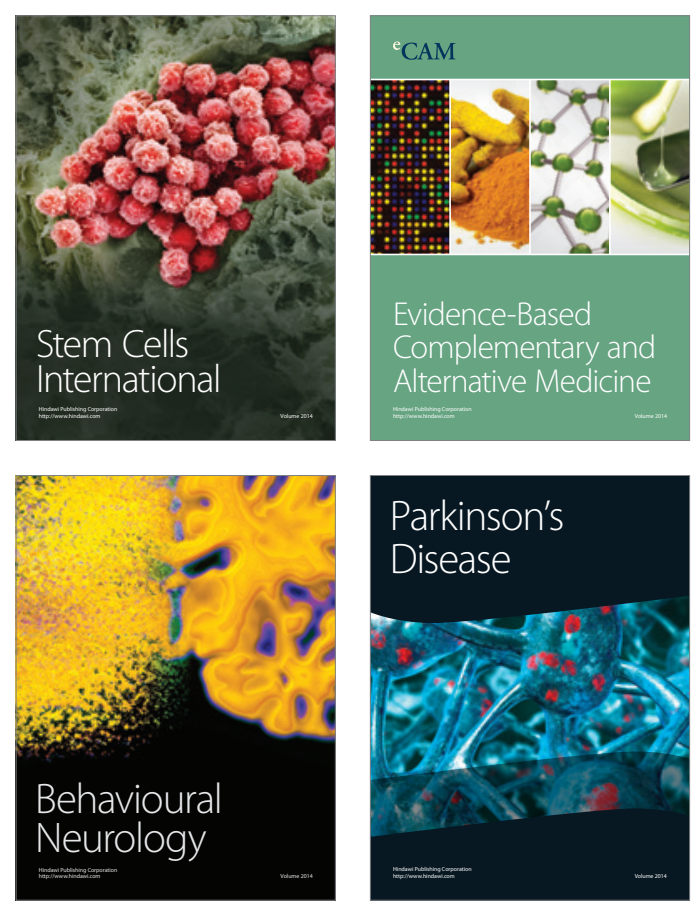

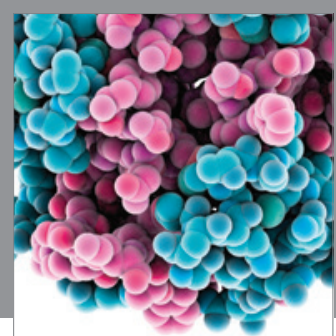

Journal of
Diabetes Research

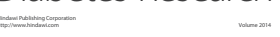

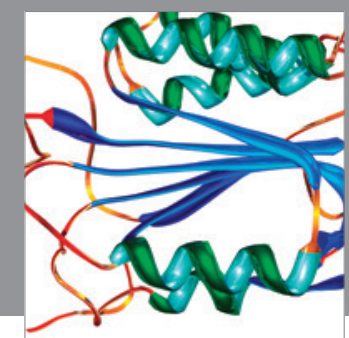

Disease Markers
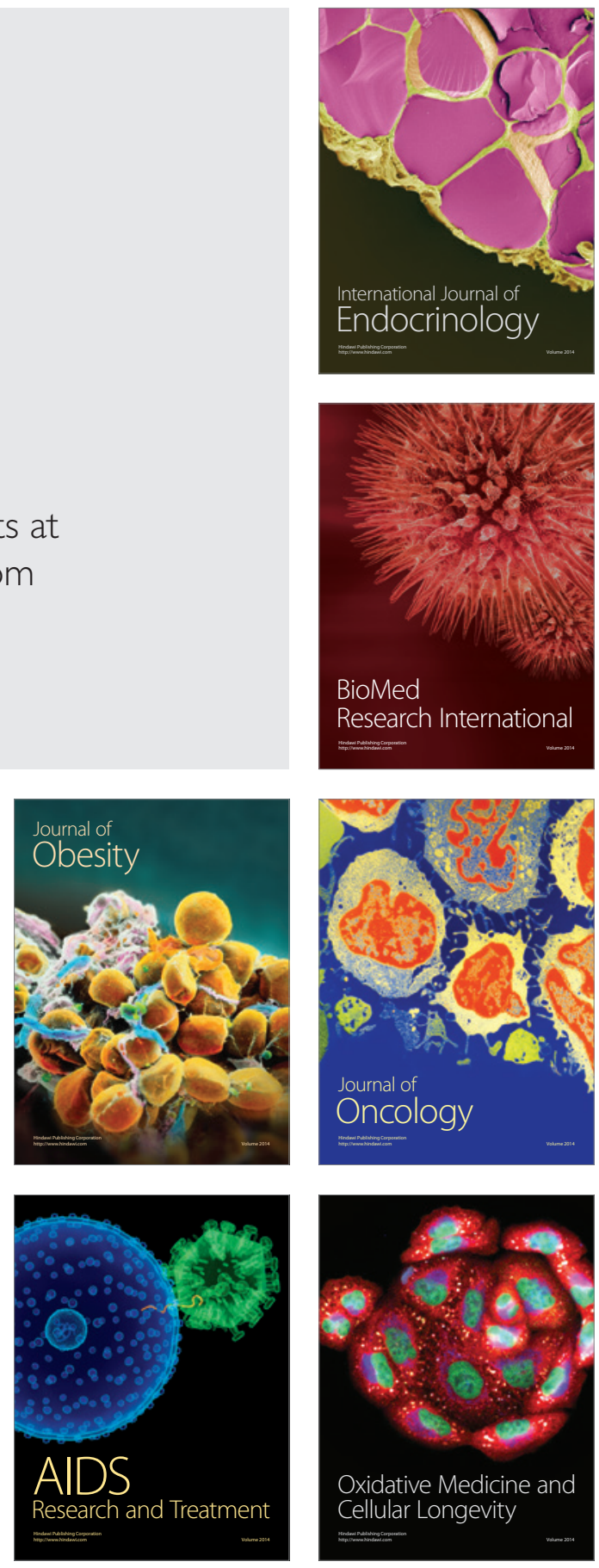